\section{Chemical Masking}

Masking and Demasking of Chemical Reactions: Theoretical Aspects and Practical Applications. By D. D. Perrin. (Chemical Analysis: a Series of Monographs on Analytical Chemistry and its Applications, Vol. 33.) Pp. $x+211$. (Wiley-Interscience: New York and London, November 1970.) $£ 6.25$.

THE masking of a chemical reaction is defined at the outset of this book as the effect whereby "the concentration of a given ion in solution may be so diminished by the addition of substances which unite with the ion to form complex salts, that an ion product sufficient to form a precipitate or cause a colour reaction is no longer obtained". This is Feigl's definition of 1936. The author goes on to comment in his introduction, "In this way without having resort to physical separations, Feigl was able to make spot-tests highly selective or even specific".

Generally speaking 'masking' has come to mean the prevention of the normal reaction of an ion upon addition of a reagent with which it reacts. by the presence of a (deliberately) added reagent with which it forms a soluble complex. Thus the prevention of normal reactions of, say, the barium ion by previous addition of sulphate ion would not normally be regarded as the 'masking' of barium ion because according to Feigl's definition, first, a complexing agent has not been used, and second, separation has occurred, that is a phase separation has occurred even though filtration has not been employed.

Much confusion exists in the literature on this, however, because some authors apparently do not regard precipitation without physical removal of the precipitate as physical separation. Thus some precipitation processes are included in this book as masking actions though clearly the author has largely avoided listing precipitating agents as masking agents. It is, however, a pity that he did not exclude such separation processes completely.

Again, however, Feigl's original definition does not quite hold because $p \mathrm{H}$ control is regarded as a masking process and so also is oxidation or reduction. In the latter two instances this appears to be a reasonable extension of Feigl's definition. The essential feature of the masking action is clearly that no physical separation is involved, that everything remains in solution when one is considering solution reactions and the like.

This is the first monograph on masking agents though very many books on spectrophotometry, complexometry and so on do give quite extensive and sometimes even monographic treatment of the subject in relation to their own topics. The author has scarcely acknowledged the existence of these. though books such as Sandell's Colorimetric Determination of Traces of Metals, to mention only one of many, have served two or three generations of students very successfully in this respect.

This book, however, is an excellent one and the author has not only done us all an excellent service in writing it, but has moreover written it extremely well. The presentation is lucid and the material is arranged very successfully according to techniques: gravimetry, titrimetry, absorption spectrophotometry, ion exchange, solvent extraction, electroanalysis, and according to applications, for example, industry and biology. The author's comment that many of the techniques described in the text have been overtaken by the evolution of more physical than chemical techniques such as neutron activation and atomic absorption analysis is not really borne out in practice because both these techniques, particularly the former, rely quite heavily on separation processes such as solvent extraction, or ion exchange in their application to real everyday analytical problems.

The use of masking agents is undoubtedly here to stay for many years to come in practically all branches of analytical science. This book is a first class one. No educational library should be without it nor should any laboratory where the analysis of metals is practised at macro, micro or trace level.

T. S. WEST

\section{Electron Optics}

Electron Beains, Lenses and Optics. Vols. 1 and 2. By A. B. El-Kareh and J. C. J. El-Kareh. Vol. 1: Pp. xiii + 411. Vol. 2: Pp. xi +320. (Academic: New York and London, September 1970.) Vol. 1: £9.20; Vol. 2: £8.15.

THE first volume of this work is largely concerned with the first-order properties of the electrostatic two-cylinder lens, the einzel lens and the round magnetic lens with symmetrical polepieces. The second volume presents a full derivation of the Scherzer formulae for the thirdorder aberration coefficients, followed by a detailed account of the spherical and chromatic aberration coefficients for the two-cylinder lens and the symmetrical magnetic lens (after Glaser). The beam-spread curve and the Airy diffraction formula are also derived and discussed briefly.

The work is narrowly conceived and its contents justify neither its title, its length nor its price. Nearly four hundred pages are taken up with tables of computed values of cardinal points and axial aberration coefficients of simple lenses. These are of somewhat doubtful value for they are derived almost exclusively from well known analytical approximations. The possibility of solving the Laplace and trajectory equations numerically is hardly mentioned, and yet numerical techniques are now almost universally adopted in serious electron optical design.

Apart from the tables of data, the space is mostly taken up with line by line mathematical derivations of the analytical formulae on which the tables are based. This represents a rather extravagant use of space in an expensively produced book. The whole enterprise suffers from a lack of balance and perspective. The correction of spherical aberration, which has long been a chief preoccupation in electron optics, is dismissed in two sentences. "Several methods have been proposed to cancel this defect; however, none have so far materialized. Therefore we shall not dwell on this particular subject." Experimental data figure little, and discussions of techniques or applications are almost entirely absent, as are numerically computed results (though Vine's computations of einzel lenses are included euroneously as experimental data).

The bibliography is practically nonexistent, on the grounds that "readily available sources fill this need". This is a serious omission in a work of any pretensions, but is typical of this disappointing book, in which the contents are so much overshadowed by what has been left out. M. E. BARNETT

\section{Radical Reactions}

Free-Radical Chain Reactions. By Earl S. Huyser. Pp. vii +387 . (Wiley-Interscience: New York and London, October 1970.) $£ 9.50$.

This book is not intended for the expert in free-radical chemistry or indeed the physical chemist whose interest is primarily in kinetics, though the latter will find many interesting examples on which to reflect. For the organic chemist who wishes to know something about the subject of free-radical chemistry this book provides a useful if somewhat idiosyncratic presentation. It is occasionally repetitious and there are some curious omissions. Some ideas are presented with little experimental evidence, and at times some of the distinctions-for example, between abstraction and displacement reactions-are a little confusing. As a whole, however, the book can be recommended to the chemists for whom it is intended, though the very high price suggests they will wish to consult it in their libraries rather than purchase a personal copy.

H. M. FREY 